\title{
Influence of granular material characteristics in the behaviour of "Bouregreg Valley" soft ground improved with stone columns
}

\author{
Noura NEHAB ${ }^{1}$, Khadija BABA ${ }^{1}$, Latifa OUADIF ${ }^{1}$, Lahcen BAHI ${ }^{1}$ \\ ${ }^{1}$ Mohammadia Engineering School, 3GIE Laboratory, Mohammed V University in Rabat, Morocco
}

\begin{abstract}
The use of finite element analysis has become widespread in geotechnical practice as means of optimizing engineering tasks; it can be easily applied to the treated areas by stone columns, which are a method of improving the soil having low geotechnical properties and likely to deform significantly under load action, by incorporating granular material (commonly called ballast) compacted by remounting passes, so they act mainly as inclusions with a higher stiffness, shear strength than the natural soil. Moreover the stone columns are highly permeable and act as vertical drains facilitating consolidation of the soft soil improving the performance of the foundation. However the characteristics of this granular material influence the behavior of soft soils treated by the stone columns technique, especially: the friction angle, the cohesion, the modular ratio and the constitutive model. The choice of the constitutive model depends on many factors but, in general, it is related to the type of analysis that we intend to perform. Numerical modeling must consider the diversity of the materials nature, the complex geometry of structures- land and the behavior of materials generally nonlinear (permanent deformation). It is a simple and effective alternative to approach the real behavior of soils reinforced by stone columns and the influence of materials characteristics, it allows settlement analysis, lateral deformation, vertical and horizontal stresses in order to understand the behavior of columns and soil. It also has the advantage of integrating the settlements of the underlying layers. This paper aims to study the mechanisms of functioning and interactions of stone columns with the surrounding ground, and vis-à-vis the various parameters characterizing the granular material "ballast" and the surrounding soil, which influence the behavior of the improved soil, The paper presents, in the first part, soil conditions and the parameters associated with columns, is then presented 3D finite element analyses, so the parametric study was carried out varying several properties especially granular material properties.
\end{abstract}

\section{Introduction}

La technique des Colonnes Ballastées consiste à incorporer dans le terrain des matériaux granulaires compactés de manière à constituer des inclusions souples, présentant des caractéristiques mécaniques élevées et un fort caractère drainant. Ils sont disposés suivant un maillage régulier et sont coiffées d'un matelas de répartition des contraintes. Elles permettent ainsi d'homogénéiser et de diminuer l'amplitude globale des tassements sous l'ouvrage. Le procédé est adapté pour traiter les sols présentant des caractéristiques mécaniques faibles à très faibles.

Le projet objet d'étude consiste à renforcer ce sol par la méthode des colonnes ballastées.
Wehr (1999) et Muir Wood et al (2000) ont étudié la performance des groupes de colonnes ballastées à l'aide de l'analyse par éléments finis.

Ce travail présente, la modélisation numérique tridimensionnelle d'une colonne isolée et d'un groupe de colonnes en prenant en compte l'influence de leur mise en place.

Les modélisations en 3D sont faites en utilisant le logiciel «Plaxis 3D Foundation». Outre l'étude de l'expansion latérale lors de la mise en place, on a analysé l'influence dans la modélisation numérique des facteurs tels que l'espacement, le nombre des colonnes ainsi que les propriétés des colonnes (la loi du comportement et l'angle de frottement). 


\section{Données du sol et caractéristiques des colonnes}

\subsection{Description de l'aire d'étude}

La vallée du Bouregreg sépare les villes de Rabat et de Salé. Elle est située sur la côte Atlantique, au Nord de Casablanca. En coordonnées géographiques, la vallée se trouve à environ $34^{\circ} 01$ en Latitude Nord et $6^{\circ} 49$ en Longitude Ouest.

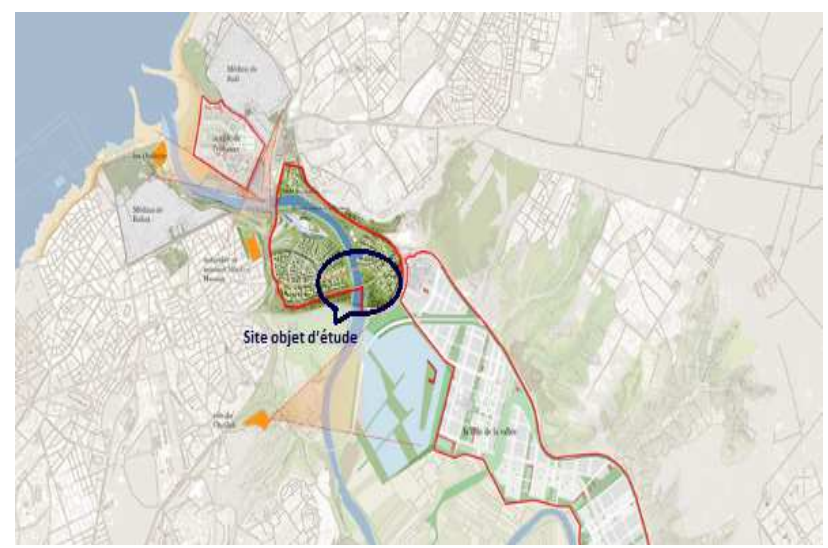

Fig.1. Géolocalisation du site.

La vallée du Bouregreg est relativement encaissée ; c'est le seul accident d'importance du relief du site de l'agglomération. Elle se développe entre les plateaux de Youssoufia Akreuch à l'Ouest et de Kariat Hssaine à l'Est, où le fleuve côtier décrit d'amples méandres. Elle se resserre près de l'embouchure et au niveau de la confluence avec l'oued Akreuch. Le barrage «Sidi Mohamed Ben Abdellah » est construit à la sortie des gorges.

La mauvaise qualité géotechnique des terrains en place contribue à majorer le risque sismique. Les versants de la vallée du Bouregreg présentent par ailleurs des signes d'instabilité par glissement de terrain ou chutes de blocs, ce qui constitue également un facteur aggravant du risque sismique.

\subsection{Les conditions géotechniques du sol et les caractéristiques des colonnes}

Une campagne de reconnaissance a été mise en œuvre au niveau du site du nouveau pont Moulay Youssef sur « oued Bouregreg ».

Des sondages carottés jusqu'à $30 \mathrm{~m}$ de profondeur, avec prélèvement d'échantillons intacts et remanié sont permis d'effectuer des essais de laboratoire (identification et caractérisation des sols) et d'établir une coupe détaillée des terrains.

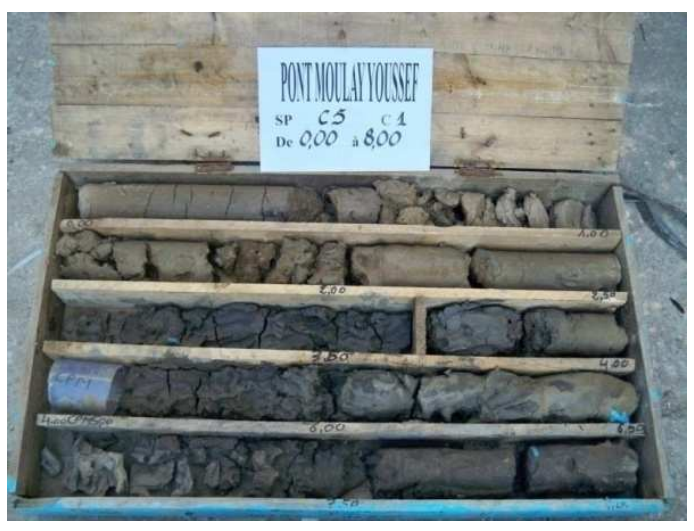

Fig.2. prélèvement d'échantillons pour effectuer les essais de laboratoire

Des sondages pressiométriques ont été réalisés avec des essais SPT afin de déterminer les caractéristiques pressiométriques des sols. En fin, l'hétérogénéité du site a été mise en évidence par des sondages de pénétration statique (CPT).

Ces essais montrent que jusqu'à $10 \mathrm{~m}$ on a affaire à des formations sableuses et vaseuses peu consistantes et qu'au-delà de cette profondeur on a du sable consistant.

Compte tenu de la granulométrie du sol et selon le règlement parasismique Marocain, celui-ci est susceptible de liquéfaction.

$\mathrm{Vu}$ le risque de liquéfaction ainsi que la présence de la vase jusqu' à $10,5 \mathrm{~m}$ de profondeur, il faut absolument traiter le sol de la rive droite par les colonnes ballastées.

Nous avons effectué une série de modélisations tridimensionnelles des colonnes ballastées avec une colonne isolée au centre et puis de groupes de colonnes, en utilisant le logiciel PLaxis 3DF.

Le sol considéré est constitué de plusieurs couches compressibles.

Le tableau suivant «Tableau 1 » présente les caractéristiques des couches du sol en place, avec

$E_{\text {oed }}=\frac{E_{M}}{\alpha}$

EM : module pressiométrique, et $\alpha$ : un coefficient rhéologique qui dépend du type du sol et de la pression limite Pl.

Table 1.Caractéristiques du sol en place.

\begin{tabular}{|c|l|l|l|l|l|}
\hline Nature du sol & $\begin{array}{l}\text { Profon } \\
\mathbf{d e u r} \\
(\mathbf{m})\end{array}$ & $\mathbf{E}_{\mathbf{M}}(\mathbf{k P a})$ & $\begin{array}{l}\mathbf{P}_{\mathbf{I}} \\
(\mathbf{k P a})\end{array}$ & $\boldsymbol{\alpha}$ & $\begin{array}{l}\mathbf{E}_{\text {oed }} \\
(\mathbf{k P a})\end{array}$ \\
\hline silts vaseux & 2 & 1700 & 200 & $1 / 2$ & 3400 \\
\hline vase & 6,5 & 2080 & 180 & $1 / 2$ & 3830 \\
\hline Vase \& sable & 10,5 & 15200 & 1476 & $2 / 3$ & 27164 \\
\hline sable fin & 17 & 18800 & 1902 & $1 / 3$ & 49122 \\
\hline
\end{tabular}




\begin{tabular}{|c|l|l|l|l|l|}
\hline & & & & & \\
\hline sable vaseux & 18 & 25000 & 2600 & $1 / 3$ & 75757 \\
\hline sable fin & 18,5 & 24000 & 2500 & $1 / 3$ & 72727 \\
\hline sable vaseux & 19,5 & 26000 & 2340 & $1 / 3$ & 52525 \\
\hline $\begin{array}{c}\text { sable fin } \\
\text { compact }\end{array}$ & $>20$ & 33840 & 3006 & $1 / 3$ & 85442 \\
\hline
\end{tabular}

Les deux figures ci-après ( fig. 4 »et «fig. 5 ») présentent les tassements déterminés à partir des deux modèles (comportement élastique linéaire et comportement élasto-plastique) pour lesquels le module de Young du ballast varie entre 40, 70 et $100 \mathrm{MPa}$,

\subsubsection{Comportement élastique linéaire :}

\section{Modélisation numérique en 2D d'une colonne isolée}

Elle permet d'assimiler le comportement de la colonne ballastée, on s'intéresse dans cette partie à l'influence des caractéristiques du matériau granulaire (Ballast) sur l'amélioration du tassement.

Une étude paramétrique a été réalisée afin de déterminer l'influence de certains paramètres du matériau granulaire sur les résultats du tassement.

Nous avons fait varier certains paramètres :

-la loi de comportement du matériau granulaire (Le modèle élastique linéaire et Le modèle élasto-plastique « Mohr-Coulomb »)

-Le module de Young du ballast (de 40 à $100 \mathrm{MPa}$ )

-L'angle de frottement du ballast (de $35^{\circ}$ à $45^{\circ}$ )

\subsection{La loi de comportement de la colonne :}

La figure «fig. 3 » présente le tassement mesuré déterminé à partir de deux modèles réalisés avec Plaxis 2D. L'un étant avec une colonne à loi de comportement élastique linéaire $($ Eballast $=70 \mathrm{MPa}) \ll \bmod$ lin elast $»$ et une colonne à loi de comportement élasto-plastique «Mohr-Coulomb $» \quad\left(\right.$ Eballast $=70 \mathrm{MPa},{ }^{\varphi}=40^{\circ}$ ) « modMohr $\mathrm{C}$ ». Les sols étant à l'état non drainé.

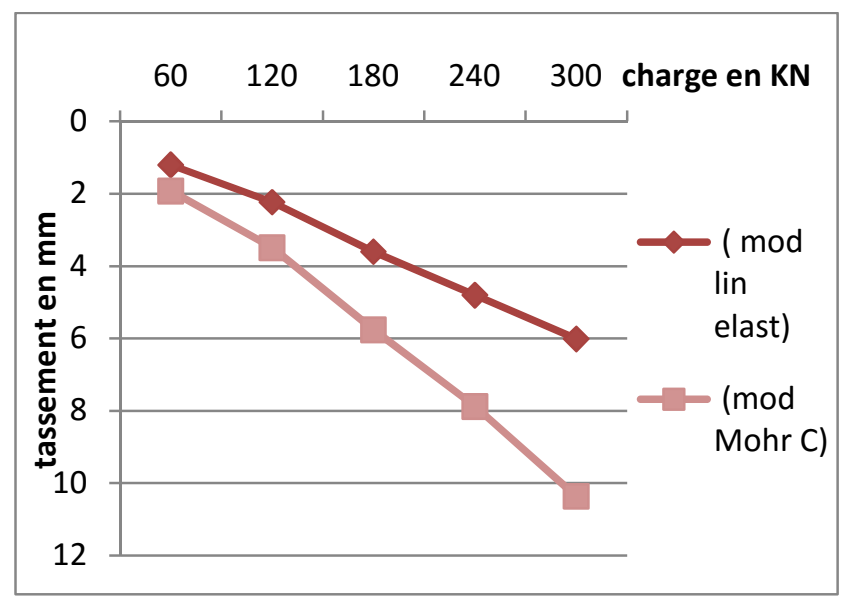

Fig.3. Résultats des tassements obtenus en 2D (colonne à loi de comportement élastique linéaire ou élasto-plastique).

\subsection{Le module de Young du ballast (de 40 à $\mathrm{MPa})$ :}

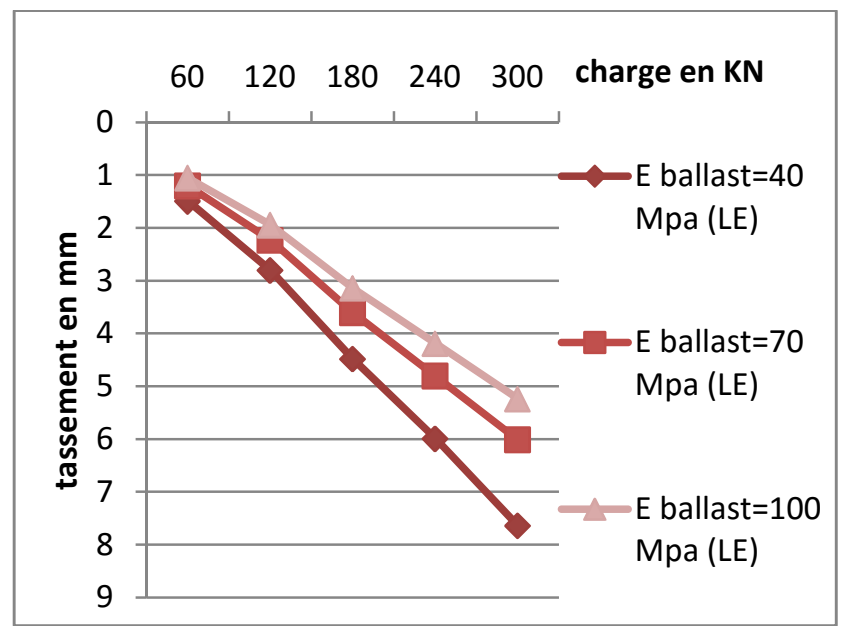

Fig. 4.Résultat des tassements obtenus en 2D (colonne à loi de comportement élastique linéaire avec $\mathrm{E}$ ballast $=40,70$ et 100 $\mathrm{MPa})$.

On constate que :

-Le modèle « $\mathrm{E}=100 \mathrm{MPa} »$ sous-estime les tassements.

-L'amélioration du sol avec l'augmentation du module de Young du ballast.

\subsection{2 comportementélasto-plastique :}

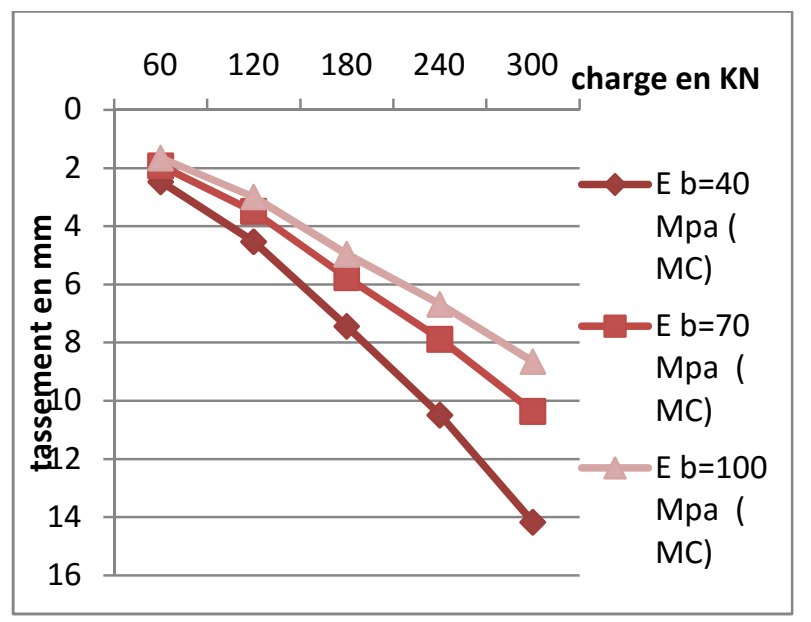

Fig. 5. Résultat des tassements obtenus en 2D (colonne à loi de comportement élasto plastique « $\mathrm{MC}$ » avec $\mathrm{E}$ ballast $=40,70$ et $100 \mathrm{MPa})$.

\subsection{L'angle de frottement du ballast (de $35^{\circ}$ à $\left.45^{\circ}\right)$ :}

La figure « fig. 6 » présente le tassement de la semelle sur la colonne ballastée, déterminé à partir du modèle réalisé 
avec une colonne à loi de comportement élasto-plastique «Mohr-Coulomb» (Eballast $=70 \mathrm{MPa}$ ) pour lequel l'angle de frottement du ballast varie de 35 à $45^{\circ}$.

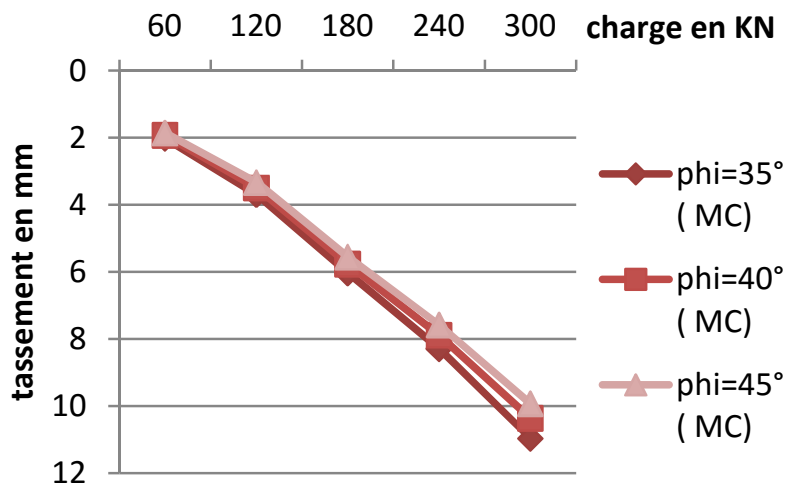

Fig. 6. Résultat des tassements obtenus en $2 \mathrm{D}$ (colonne à loi de comportement élasto plastique « $\mathrm{MC} », \mathrm{E}$ ballast et $\varphi=35^{\circ}, 40^{\circ}$ et $45^{\circ}$ ).

On constate que l'augmentation de l'angle de frottement interne du ballast permet une réduction de tassement.

\section{Modélisation numérique en 3D d'une colonne isolée}

Cette section analyse l'amélioration du tassement, pour le cas d'une colonne isolée, cette amélioration va être déterminée par le facteur de réduction d tassement, $\beta$.

Les paramètres à varier sont :

- L'angle du frottement interne du matériau granulaire.

- La loi du comportement du matériau granulaire

\subsection{L'angle du frottement du ballast}

Pour examiner l'influence de L'angle du frottement interne du matériau granulaire, et le rapport des surfaces pour une colonne isolée, on prend comme loi du comportement de la colonne « la loi Mohr coulomb».

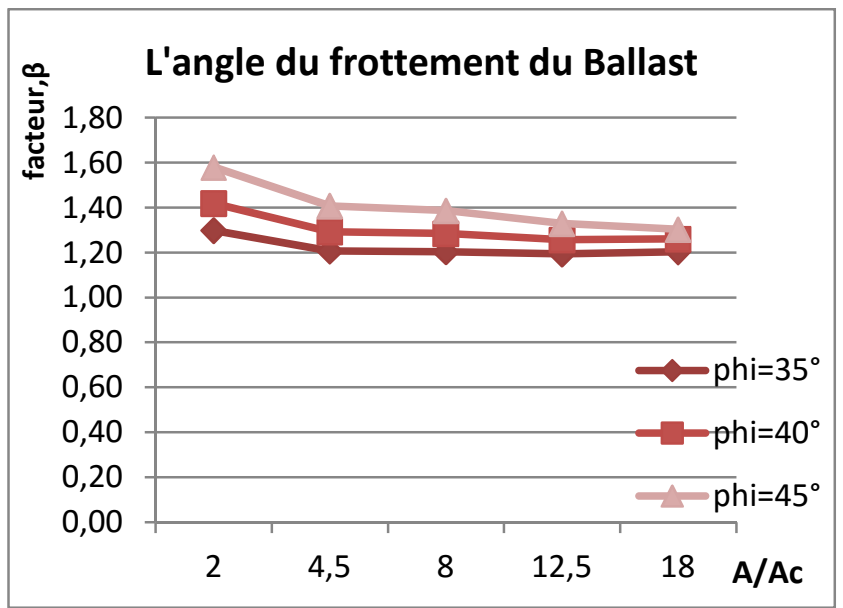

Fig. 7. L' influence de l' angle de frottement interne du ballast et du rapport des surfaces $\mathrm{A} / \mathrm{Ac}$.

Les graphes (Figure 7) illustre pour les différentes valeurs de $\mathrm{A} / \mathrm{Ac}$, le facteur de réduction du tassement, $\beta$ pour les différentes valeurs de $\Phi\left(35^{\circ} / 40^{\circ} / 45^{\circ}\right)$ :

$\Leftrightarrow$ on observe que l'augmentation de l'angle de frottement interne $\mathrm{du}$ ballast permet une réduction de tassement.

$\Leftrightarrow$ pour $\mathrm{A} / \mathrm{Ac}=2$ (petites valeurs du rapport des surfaces); $\beta$ varie de $1.30\left(\Phi=35^{\circ}\right)$ à $1.58(\Phi$ $=45^{\circ}$ ), alors que pour $\mathrm{A} / \mathrm{Ac}=18$ (grandes valeurs du rapport des surfaces); $\beta$ varie de 1.20 ( $\Phi$ $\left.=35^{\circ}\right)$ à $1.30\left(\Phi=45^{\circ}\right)$.

$\stackrel{4}{\rightarrow}$ Le tassement augmente lorsque A/Ac augmente pour toute les valeurs de l'angle de frottement interne du ballast.

$\leftrightarrow$ Les graphes s'approchent pour les grandes valeurs de A/Ac, donc l'augmentation de l'angle de frottement interne du ballast a un effet négligeable pour les grandes valeurs du rapport des surfaces.

\subsection{La loi du comportement du ballast}

Pour examiner l'influence de La loi du comportement du matériau granulaire, et le rapport des surfaces pour une colonne isolée, on prend comme loi du comportement du sol « la loi Mohr coulomb » et $\phi=45^{\circ}$ pour la colonne (MC and HS).

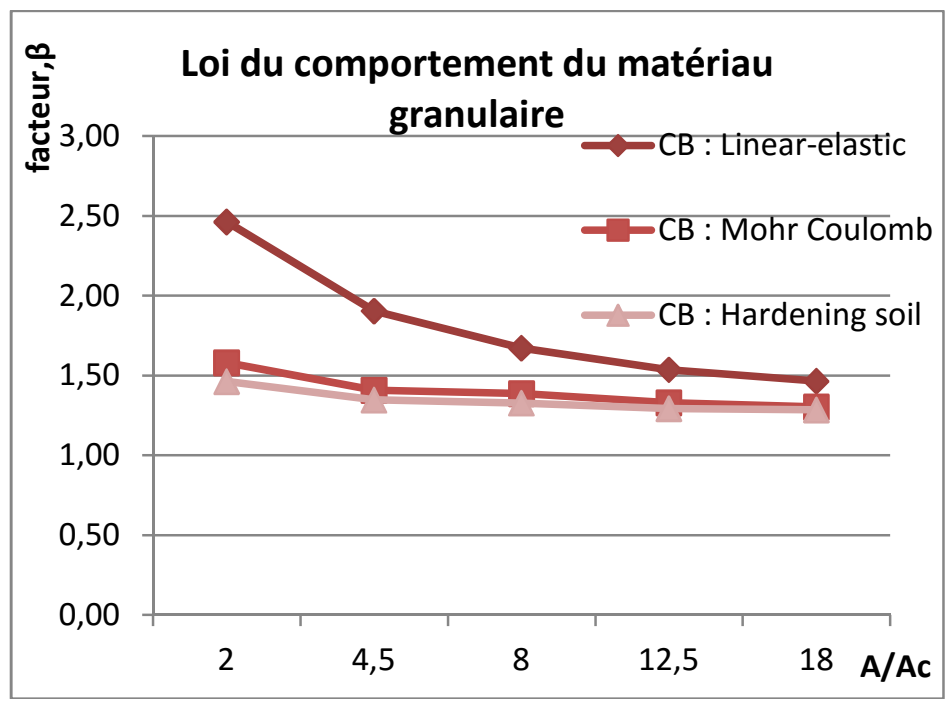

Fig. 8. L'amélioration du tassement pour les différentes lois du comportement du matériau granulaire de la colonne.

Les graphes (Figure 8) illustre pour les différentes valeurs de $\mathrm{A} / \mathrm{Ac}$, le facteur de réduction du tassement, $\beta$ pour les différentes lois de comportement de la colonne (Mohr coulomb, Hardening soil et linéaire élastique): 
$\Leftrightarrow$ Le facteur de réduction du tassement diminue avec l'augmentation de A/Ac.

$\Leftrightarrow$ Lorsqu'on varie $\mathrm{A} / \mathrm{Ac}$ de 2 à $18 ; \beta$ varie de 2.46 à 1.46 (pour le modèle linéaire élastique), de 1.46 à 1.28 (pour le modèle Hardening soil) et de 1.58 à 1.30 (pour le modèle Mohr coulomb).

$\Leftrightarrow$ Les deux lois de comportement de la colonne (Mohr coulomb et Hardening soil) atteignent approximativement le même graphe.

$\Leftrightarrow$ Les solutions élastiques surestiment le facteur de réduction du tassement.

\section{Modélisation numérique en 3D d'un groupe de 3 colonnes}

\subsection{L'influence de la position des colonnes;}

Cette étude paramétrique a pour objectif de montrer l'influence de l'espacement entre colonnes, ou plus précisément, la position relative des colonnes. On considère un groupe de trois colonnes (maille triangulaire), Le chargement est appliqué sur un carré de cote $\mathrm{B}=8 \mathrm{~m}$ et l'espacement entre les colonnes est variée de $\mathrm{s}=1$ à $4 \mathrm{~m}$.
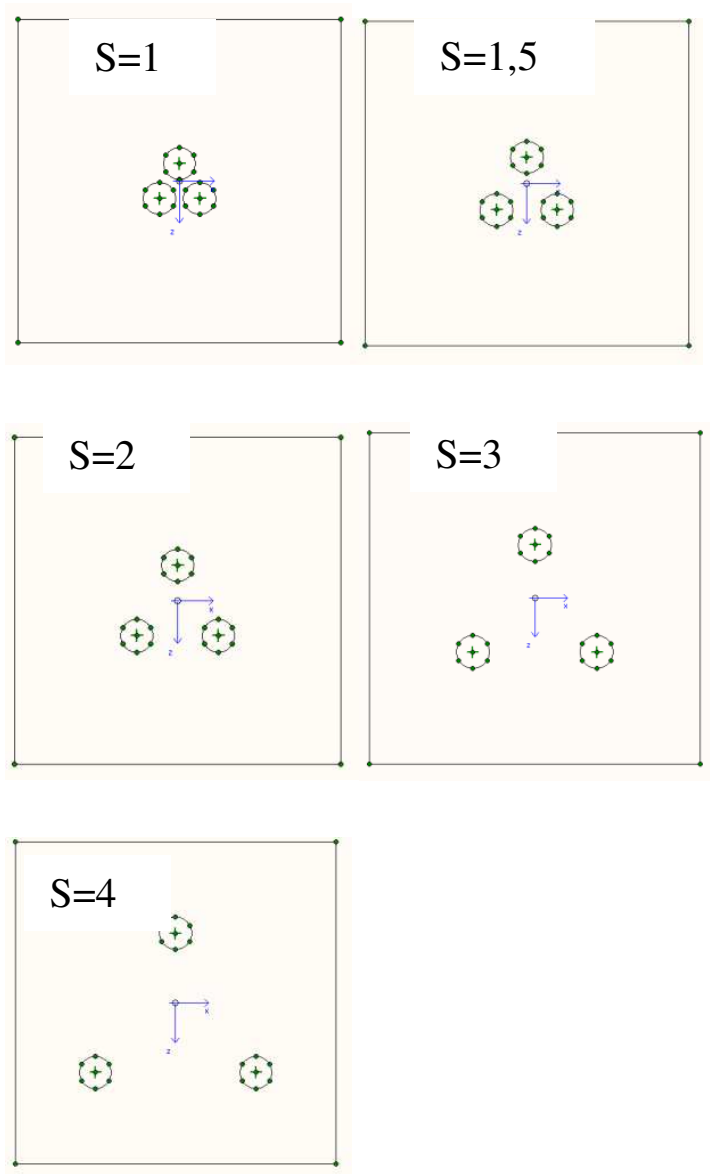

Fig. 9.La variation de l'espacement entre les colonnes pour une maille triangulaire.

5.1.1. Variation de la loi du comportement des colonnes :

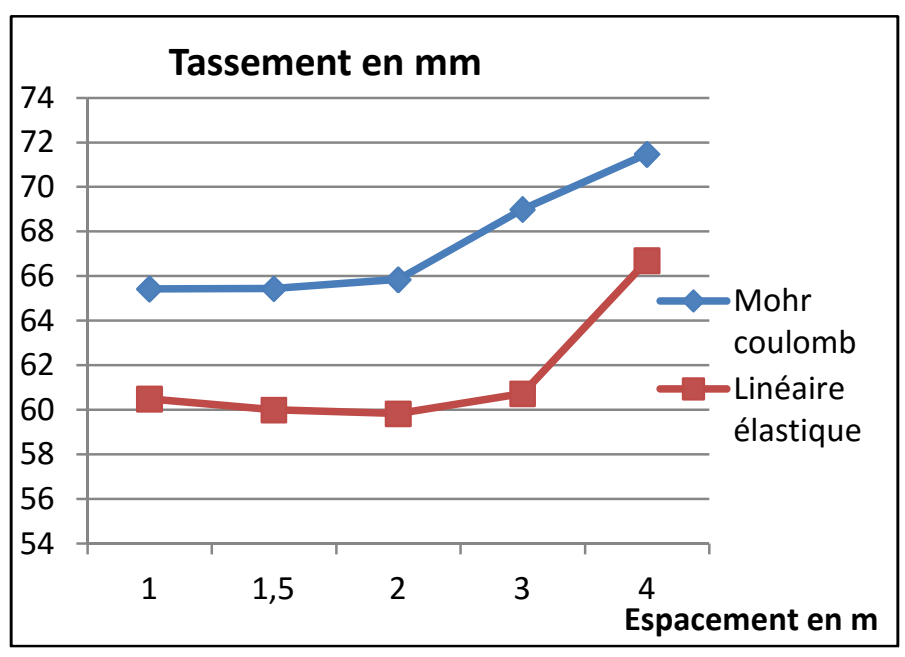

Fig. 10.L'effet de la variation de l'espacement entre les colonnes sur le tassement pour les deux lois de comportement de colonnes (MC et LE).

Le tassement augmente lorsque les colonnes sont plus espacées pour la loi de comportement Mohr coulomb. Alors que pour la loi de comportement linéaire élastique le tassement diminue légèrement entre $S=1$ et $S=2$ (mais reste très proche d'une même valeur), puis commence à augmenter.

On constate qu'à partir d'une valeur d'entraxe le tassement augmente d'une manière excessive, donc à partir de cette valeur le renforcement n'est plus bénéfique.

Ces courbes confirment la règle pratique qui recommande un entraxe de colonnes inférieur à $4 \mathrm{Dc}$ ici $4 \times 0,8=3,2 \mathrm{~m}$ ) pour obtenir un renforcement significatif.

\subsubsection{Variation de l'angle de frottement des colonnes :}

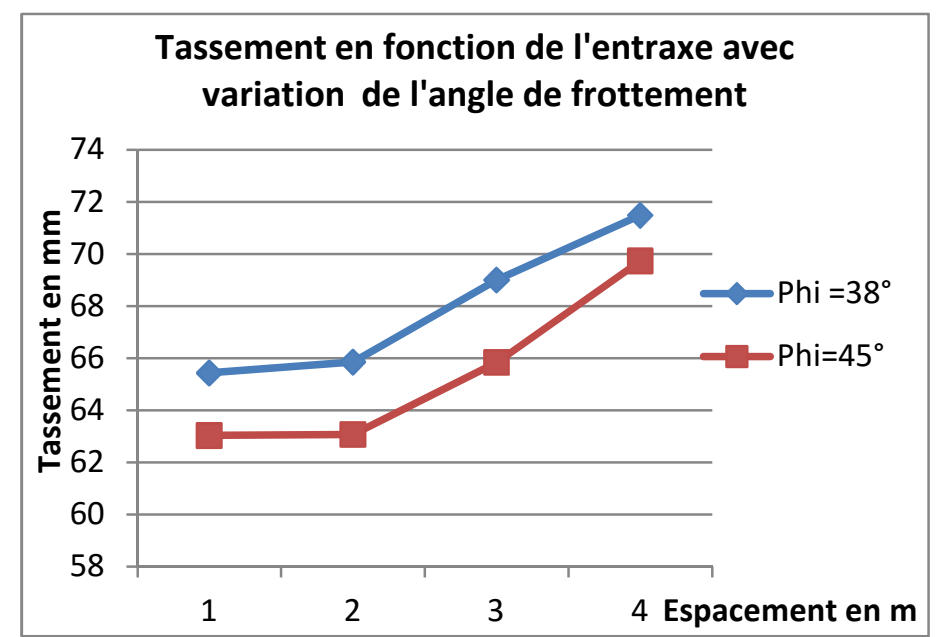


Fig. 11.L'effet de la variation de l'espacement entre les colonnes sur le tassement pour les deux angles de frottement $\left(\right.$ phi $=38^{\circ}$ et phi $\left.=45^{\circ}\right)$.

On constate que l'augmentation de l'angle de frottement interne du ballast permet une réduction de tassement. De même Le tassement augmente lorsque les colonnes sont plus espacées pour les deux valeurs d'angle de frottement interne du ballast.

\subsection{L'influence du nombre des colonnes;}

Dans cette étude on a utilisé un maillage carré en changent le nombre de colonnes de 1 à 16 colonnes, ainsi que la surface de chargement pour les deux lois de comportement des colonnes Mohr coulomb et linéaire élastique, on a gardé un espacement de 1,5 m entre les colonnes, et un angle de frottement de $38^{\circ}$.

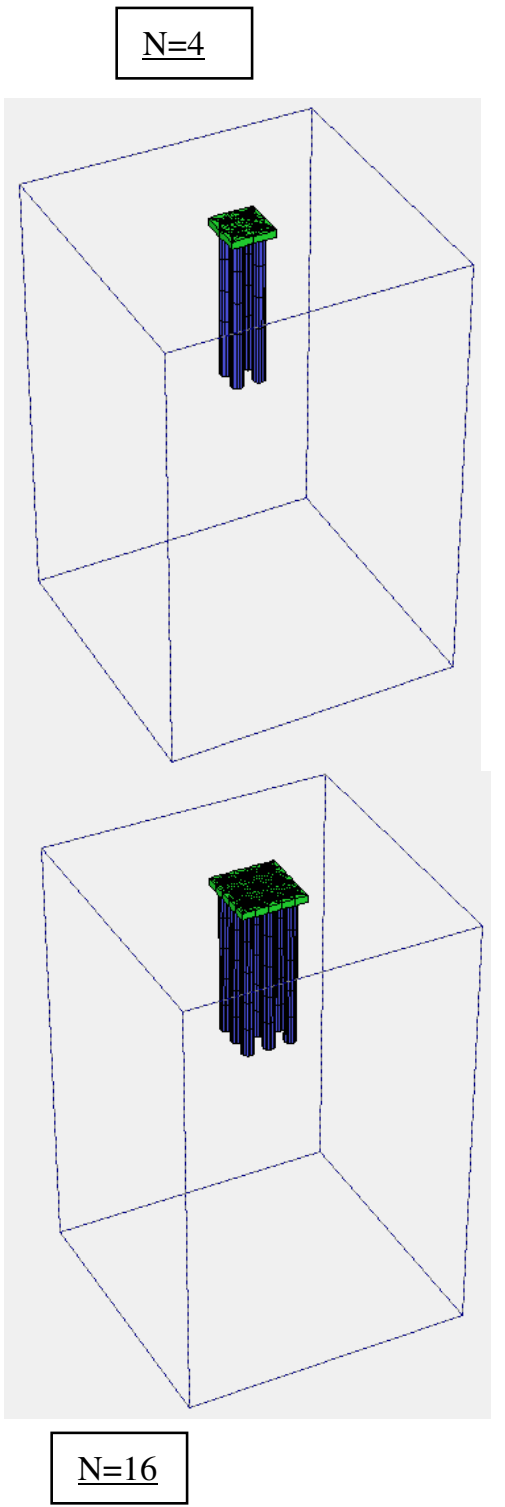

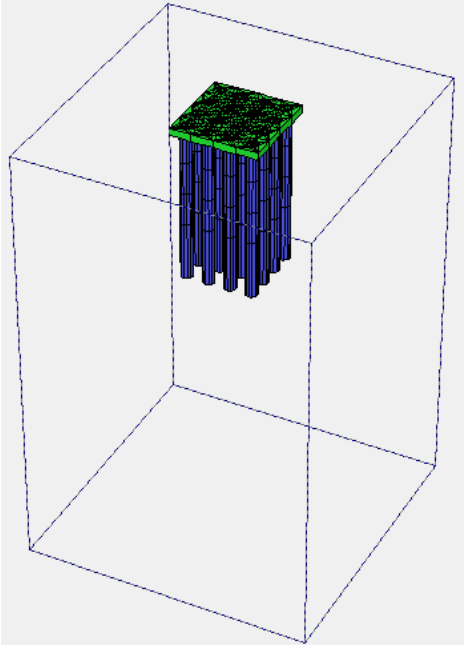

Fig. 12.La variation du nombre des colonnes pour ne maille rectangulaire

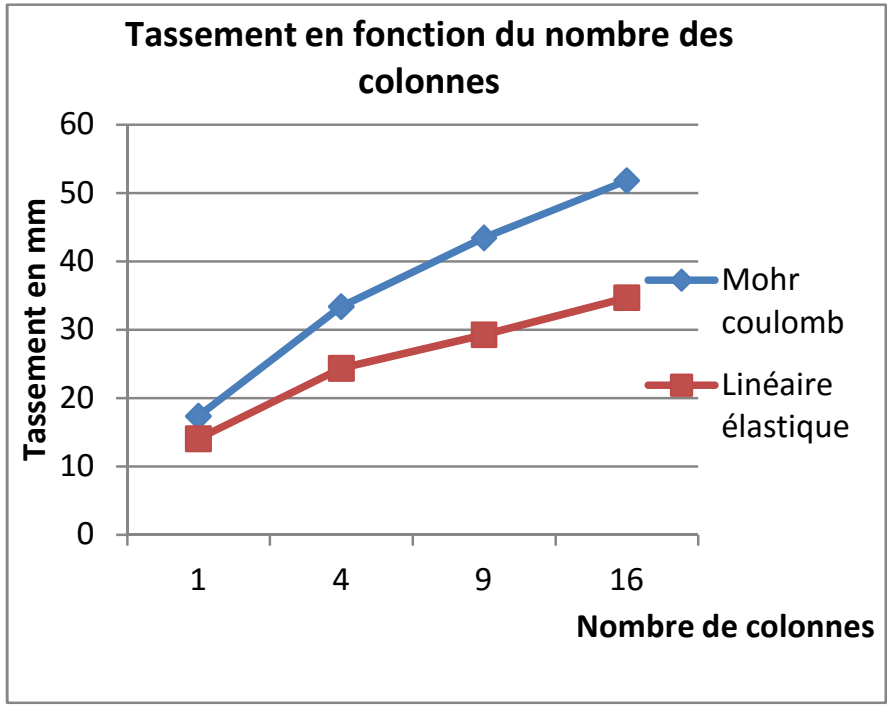

Fig. 13.L'effet de la variation du nombre des colonnes sur le tassement pour les deux lois de comportement de colonnes (MC et LE).

-Les deux courbes se rapprochent dans le cas d'un petit nombre de colonnes et se divergent dans le cas contraire. -Le nombre de colonnes n'a pas une grande influence sur les tassements, différemment de la littérature qui confirme qu'un nombre croissant de colonnes est considéré comme ayant un effet bénéfique parce que les colonnes sont mieux confinées latéralement [Priebe].

\section{Conclusion :}

Nous avons effectué une série de modélisations tridimensionnelles des colonnes ballastées avec une colonne isolée au centre, en utilisant le logiciel PLaxis 3DF. Le sol considéré est constitué de plusieurs couches compressibles. 
L'augmentation des propriétés du matériau granulaire (l'angle de frottement, le raport modulaire) permet d'améliorer le sol traité en augmentant le facteur de réduction du tassement, l'influence de ces paramètres est liée à l'arrangement des colonnes (la position des colonnes), et à la loi de comportement adopté pour la colonne.

Pour un groupe de trois colonnes, disposées aux sommets d'un triangle équilatéral. La modélisation en 3D permet de montrer que l'espacement entre les colonnes augmente le tassement pour les deux lois de comportement, et à partir d'une valeur d'entraxe le tassement augmente d'une manière excessive et le renforcement n'est alors plus bénéfique. Alors que le nombre de colonnes n'influence pas beaucoup sur les résultats.

\section{References}

[1] Wehr J. (2004). Stone columns- single columns and group behavior. In proceedings of the 5th Intconfon Ground Improvement techniques. Malaysia, 329-340.

[2] Wehr, W. (1999). Schottersaulen - das verhalten von einzelnensaulen und saulengruppen.Geotechnik, vol.22,n ${ }^{\circ}, 40-47$.

[3]Killeen M. (2012). Numerical modeling of small groups of stone columns. PhD Thesis. National university of Ireland, Galway.

[4]Brinkgreve, R.B.J., Engin, E. and Swolfs, WM. (2012). Plaxis 3D 2012 Manual.Plaxisbv, the Netherlands.

[5]Baumann V. and Bauer G. E. A. (1974). The performance of foundations on various soils stabilized by the Vibrocompaction process, Rev.canadienne de Géotechnique, Vol.11, n², nov.1974, 509-530.

[6] Black, J.V., Sivakumar, V. and McKinley, J.D. (2007). Performance of clay samples reinforced with vertical granular columns. Canadian Geotechnical Journal 44, pp.89-95.

[7]Hughes, J.M.O. and Withers, N.J. (1974). Reinforcing of soft cohesive soils with stone columns.Ground Engineering 7 (3), 42-49.

[8]McKelvey, D., Sivakumar, V., Bell A. and Graham J. (2004). Modeling vibrated stone columns in soft clay.

[9]Proceedings of the Institute of Civil Engineers Geotechnical Engineering 157(3), 137-149.

[10] Muir Wood, D., Hu, W. and Nash, D.F.T. (2000). Group effects in stone column foundations model tests.Géotechnique 50 (6), 689-698.

[11]Najjar, S.S., Sadek, S. and Maakaroun, T. (2010). Effect of sand columns on the undrained load response of soft clays.Journal of Geotechnical and Geoenvironmental Engineering 136(9), 1263-1277.

[12]Nehab, N., Baba, K., Ouadif L., Cherradi C. and Bahi L. (2014). Soft soil strengthening by stone columns: case of the embankment under the bridge "Moulay Youssef" (Rabat/Salé). MATEC Web of Conferences.MatecconfDOI: 10.1051//20141102012.

[13]Shahu, J. and Reddy, Y. (2011). Clayey Soil Reinforced with Stone Column Group: Model Tests and Analyses.

[14]Slocombe, B.C. (2001). Deep compaction of problematic soils, Problematic Soils, Thomas Telford (London), 163-181.

[15]Priebe H., (1976). Abschätzung des SetzungsverhalteseinesdurchStopfverdichtungverbesserte nBaugrundes, Die Bautechnik, Vol. 53 (1976), n 5, 160162.

[16]Ghionna, V., Jamiolkowski, M. (1981). Colonne di ghiaia. X Ciclo di conferenze dedicate aiproblemi di meccanicadeiterreni e ingegneriadellefondazionimetodi di miglioramento deiterreni, Politecnico di Torino Ingegneria, attidell'istituto di scienza delle costruzioni, $\mathrm{n}^{\circ}$ 507, nov. 1981.

[17]Khabbazian, M., Kaliakin, V. N. and Meehan, C. L. (2012). Numerical Simulation of Column Supported Embankments with Geosynthetic Encased Columns: Influence of Soft Soil Constitutive Model. Conference: GeoCongress 2012. DOI: 10.1061/9780784412121.001

[18]Guetif, T., Bouassida, M. and Debats, J. M. (2007). Improved soft clay characteristics due to stone column installation. Computers and Geotechniques, 34, 104-111. 